\title{
Magnetic resonance imaging of juxtapapillary plaques in cadaver eyes ${ }^{\star}$
}

\author{
David F Williams, William F Mieler, Glenn J Jaffe, Dennis M Robertson, Lloyd Hendrix
}

\begin{abstract}
Adequate treatment of juxtapapillary melanomas with episcleral plaque brachytherapy using lower energy radiation sources may be difficult because of uncertainties regarding the relationship of the plaque to the optic nerve and tumour base. We obtained magnetic resonance images of a dummy plaque placed in a juxtapapillary location in cadaver specimens. Although it is possible to place a plaque in close association with the optic nerve sheath, a tissue barrier exists which may prevent actual contact between the plaque and nerve. Posterior tilting of the plaque may also occur. Because of these uncertainties regarding plaque placement, juxtapapillary melanomas should be considered a distinct subgroup when evaluating the efficacy of radioactive plaque brachytherapy in the treatment of choroidal melanoma.
\end{abstract}

Juxtapapillary melanomas (those extending within $3 \mathrm{~mm}$ of the optic nerve head) constitute approximately 3 to $33 \%$ of tumours in reported series of radiation treated choroidal melanomas. ${ }^{1-3}$ When giving radioactive plaque therapy in the treatment of juxtapapillary choroidal melanomas several problems are encountered that are not seen in the treatment of more peripherally located tumours. First, the diameter of the optic nerve sheath is 1.5 to $2.5 \mathrm{~mm}$ larger than the optic disc. ${ }^{4}$ Thus when treating a tumour in apposition with the disc it is not possible to place a plaque fully round the base of the tumour. Secondly, it is not clearly known how close to the optic nerve sheath it is possible to place a plaque owing to uncertainties in the posterior anatomy. Thirdly, tilting of the plaque away from the globe may create further uncertainty in the plaque relationship to the optic nerve and the adjacent sclera, such that accurate estimates of radiation delivered to the tumour and/or optic nerve may not be possible. Because of these uncertainties in plaque placement, the ability adequately to treat tumours whose posterior border is within 2 to $3 \mathrm{~mm}$ of the optic nerve head may be questioned.

We used magnetic resonance imaging to investigate the relationship of a plaque to the

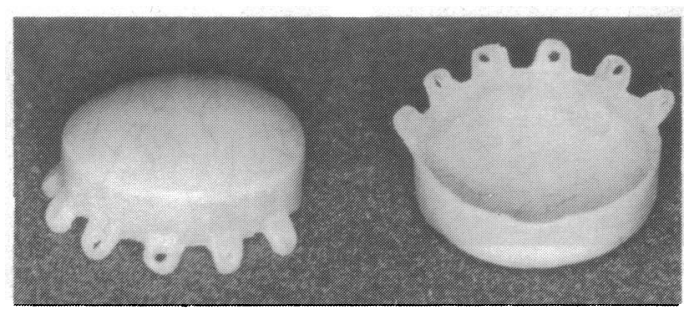

Figure 1: Plastic dummy plaque. optic nerve in six fresh unembalmed cadaver specimens 12-72 hours post mortem. The purpose of this study was to evaluate the placement of a plaque with regard to the optic nerve sheath in the different quadrants of the globe, and to establish whether tilting of the plaque away from the sclera may occur, or other positioning problems as well.

\section{Material and methods}

A plastic dummy plaque, identical in design to the Collaborative Ocular Melanoma Study (COMS) plaque 5 measuring $14 \mathrm{~mm}$ in diameter with a $2.6 \mathrm{~mm}$ circumferential lip was used (Fig 1). The plaque was placed in a juxtapapillary location in all four quadrants of the globe in six eyes from fresh cadaver specimens. After the conjunctiva and Tenon's capsule had been incised, the plaque was placed on the scleral surface. The globe was rotated away from the desired quadrant by rectus muscle sutures, and the plaque was slid on the scleral surface between the recti muscles as far posteriorly as possible. When significant resistance to further movement was encountered, and when the entire globe moved with further attempts at posterior positioning, apposition to the optic nerve sheath was presumed and manipulation was stopped. The globe was then rotated back to the primary position while mild posteriorly oriented pressure was maintained on the plaque to prevent plaque movement. In all instances the anterior position of the plaque could be directly observed and was noted to be in apposition to the sclera; therefore the plaque was not sutured to the sclera. The conjunctiva and Tenon's capsule were then reapproximated loosely to the limbus.

Magnetic resonance images were obtained

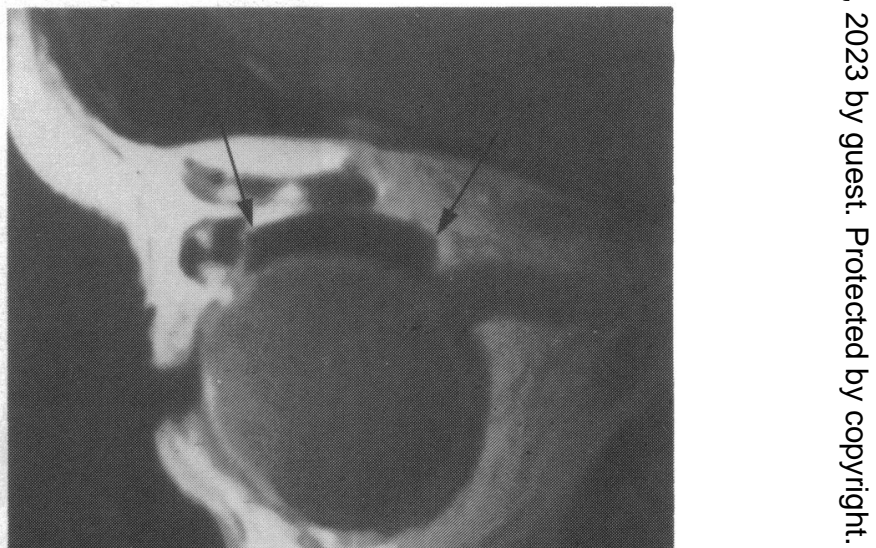




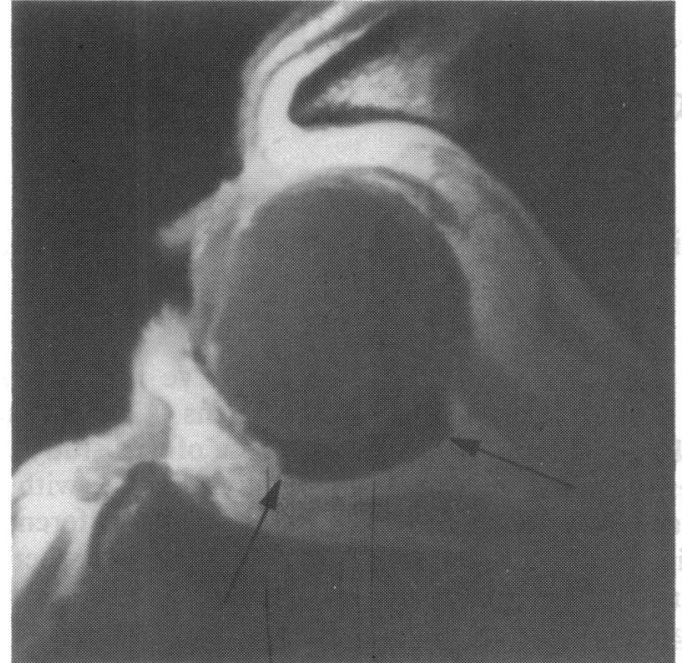

Figure 2B: Right, plaque in the inferotemporal quadrant (arrows.)

with a General Electric Sigma system operating at 1.5 tesla. A 2 -inch $(5-\mathrm{cm})$ diameter counterrotating current surface coil $^{6}$ (Medical Advances, Inc, Wauwatosa, WI) was used as a local radio frequency receiver and the body coil as a transmitter. Axial, sagittal, and oblique $\mathrm{T} 1$ weighted images $\left(T_{R}=600 \mathrm{~ms}, T_{E}=20 \mathrm{~ms}\right)$ were obtained. Additional parameters included slice thickness of $3 \mathrm{~mm}$ with a gap of $1 \mathrm{~mm}, 8 \mathrm{~cm}$ field of view, $256 \times 128$ matrix, and two excitations. Acquisition time averaged 4 minutes for a series of eight images. The plaque, which contains no mobile protons, emits no signal and therefore appears black in contrast to the surrounding soft tissues.

\section{Results}

We obtained images showing the plaque relationship to the optic nerve in the four quadrants of the globe. A total of six eyes were scanned. The plaque could be placed in close association with the optic nerve sheath in all four quadrants. A close approach to the optic nerve sheath was easily obtained superonasally and inferonasally (Figs 2A, B) while the infero-

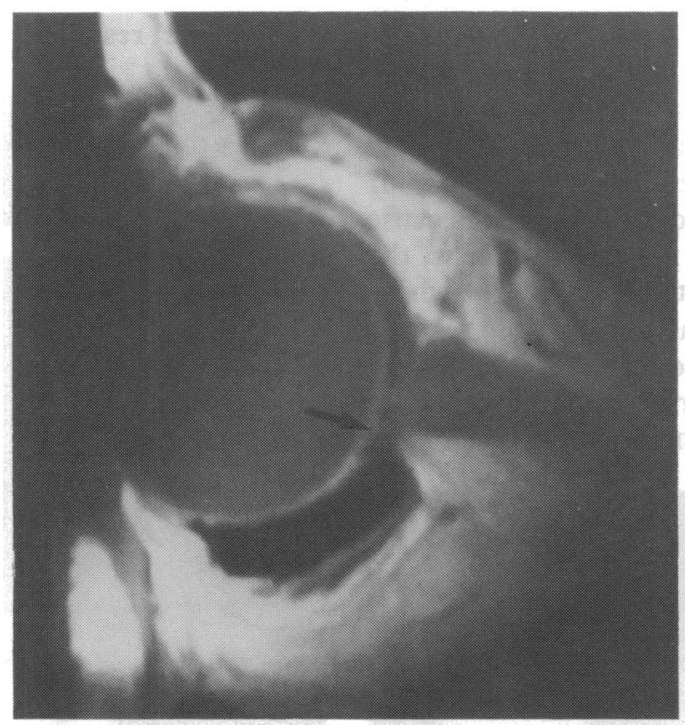

Figure 3: Magnetic resonance image of plaque in the inferotemporal quadrant demonstrating a tissue barrier between the plaque and optic nerve sheath (arrow).

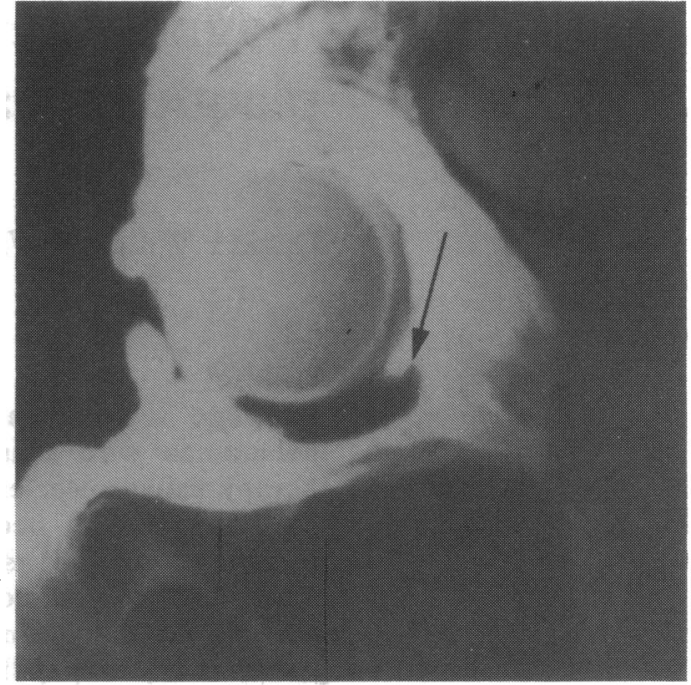

Figure 4: Tilting of posterior portion of plaque away from the sclera (arrow).

temporal approach required slightly more manipulation and the superotemporal approach was the most difficult.

In several scans in which the plaque was thought to be in apposition to the optic nerve the scan showed tissue between the plaque and the optic nerve (Fig 3).

Additional difficulties encountered in plaque placement were tilting of the posterior portion of the plaque away from the optic nerve and sclera (Fig 4), and posterior positioning of the plaque along the optic nerve sheath (Fig 5). These findings each occurred in one of six eyes scanned with plaque placement in the inferior quadrants.

\section{Discussion}

The rationale for the treatment of ocular melanoma with radioactive plaque brachytherapy is the delivery of an amount of radiation adequate to induce regression of the tumour while minimising radiation scatter to adjacent ocular tissues, thus preserving visual function. Optimal treatment of the tumour requires that the radiation field fully encompass all borders of the tumour. The use of high energy radiation sources, such as cobalt 60 , results in significant scatter of radiation from which it is not possible adequately to shield adjacent tissues. This scattered radiation may minimise undertreatment of

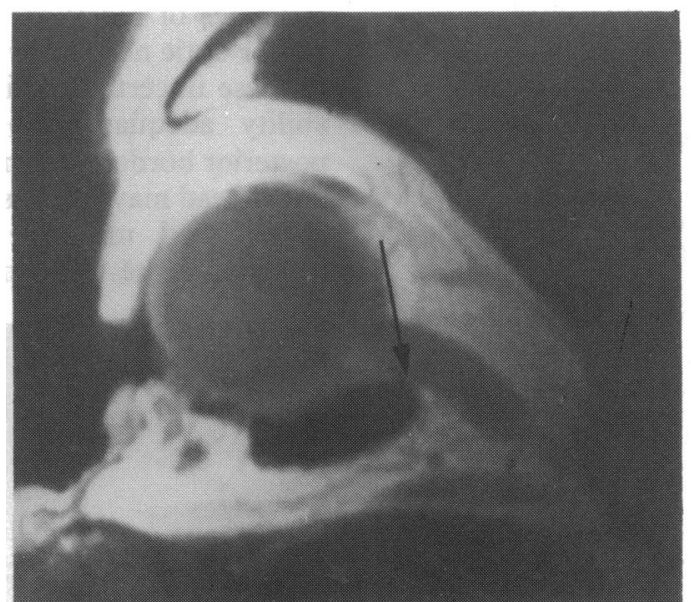

Figure 5: Migration of plaque along the optic nerve (arrow). 
tumour margins when a plaque is not accurately placed over the tumour base but may also result in excessive radiation damage to normal ocular tissues. Radiation scatter may be minimised by utilising $\beta$ emitters or low energy $\gamma$ emitters, such as ruthenium-106 or iodine-125, and by providing a circumferential lip on the plaque to shield adjacent tissues. ${ }^{17-9}$

Brachytherapy treatment of juxtapapillary melanomas with low energy radiation sources presents special difficulties because of uncertainties in the posterior anatomy with regard to plaque placement. It is known that the optic nerve within the scleral canal is approximately $1.5 \mathrm{~mm}$ in diameter while the optic nerve sheati is $3-4 \mathrm{~mm}$ in diameter. ${ }^{4}$ Thus, even if a plaque were placed in apposition to the optic nerve it may not be possible fully to cover the base of a tumour with a margin extending closer than 0.75 to $1.25 \mathrm{~mm}$ from the optic nerve head. If the plaque is not placed in apposition to the optic nerve sheath, the potential for adequate treatment of a juxtapapillary melanoma would diminish accordingly. Echography has been used to verify radioactive plaque positioning in the treatment of choroidal melanoma. ${ }^{1011}$ The assessment of the position of the plaque with regard to the optic nerve is quite difficult, however, owing to the acoustic shadowing caused by the strong reflectance of the metallic plaque.

Using magnetic resonance imaging, we have obtained clear images and have shown that it is possible to place a plaque in close association with the optic nerve sheath. However, an anatomical barrier does exist which may prevent actual contact between the plaque edge and the optic nerve (Fig 2). Although the resolution obtained is insufficient precisely to identify the small anatomical structures, it is likely that the penetration of the short posterior ciliary arteries and nerves circumferential to the optic nerve provide at least part of the anatomical barrier between the plaque and nerve.

We also found that more manipulation was required to place the plaque adjacent to the optic nerve in the temporal quadrants than in the nasal quadrants. Potential anatomical factors making the temporal approach to the optic nerve more difficult include the insertion of the inferior oblique muscle and the greater distance from the temporal limbus to the optic nerve.

Another difficulty which may be encountered is tilting of the plaque away from the sclera. Because of the relative inaccessibility of the point of insertion of the optic nerve into the sclera, direct observation of the most posterior portion of the plaque may not be possible, and posterior tilting of the plaque may occur unknown to the surgeon. The radiation source would thus be displaced away from the tumour apex and directed towards the optic nerve, with resultant under treatment of the tumour and excessive radiation scatter to the nerve.

There are several potential complicating factors in this study. (1) We used unembalmed cadaver specimens which were 12 to 72 hours old. The effect of postmortem tissue changes on ease of plaque placement and the ability to obtain apposition to the optic nerve are unknown. (2)
All scans were obtained with the specimen in the supine position. The effect of different body positions or ocular movements on plaque-nerve orientation are unknown. (3) Our dummy plaque was made from lightweight plastic. A heavier metallic plaque with radioactive seeds may result in changes in plaque-nerve orientation, particularly with patient movement. (4) The circumferential lip on the dummy plaque was $2.6 \mathrm{~mm}$. It is possible that, if the lip was smaller, thus producing a 'flatter' plaque, there would be less tissue distortion and less resistance to posterior placement of the plaque. This smaller lip would result in less shielding of scatter radiation, however. In a similar fashion, a notched plaque could be utilised, though there is still potential for tissue distortion and it may not be possible to place the plaque in true apposition with the optic nerve sheath. (5) the rectus and oblique muscles were not detached, and the ease of application of a plaque directly under the muscles is not fully known. Though posterior visualisation would be enhanced with muscle removal, direct assessment of the optic nerve sheath remains exceedingly difficult. And, finally (6), technical difficulties involving image acquisition on the magnetic resonance scanner in an oblique plane may significantly affect image quality and acquisition time. It is expected that as advances in software occur, oblique images will become easier to obtain and the images will be of improved quality.

In summary, low energy brachytherapy treatment of a juxtapapillary melanoma may be inadequate for several reasons. The normal anatomical relationship of the optic nerve sheath to the optic nerve head places a limit on how close a tumour margin can be to the optic nerve head and still be potentially covered by an episcleral plaque. A tissue barrier between the plaque rim and optic nerve sheath, as described above, may increase the difficulty of fully treating tumour margins. Tilting of the plaque may also occur, resulting in inadequate radiation delivery to the tumour apex while delivering excessive radiation scatter to the optic nerve.

Though magnetic resonance imaging cannot be readily used with metallic plaques in patients, this study demonstrates that the juxtapapillary placement of plaques may be much less precise than previously realised. These uncertainties indicate that juxtapapillary tumours should be considered as a distinct subgroup meriting separate analysis of the efficacy of treatment. This work was supported in part by an unrestricted grant from
Research to Prevent Blindness, Inc, and by National Institutes of Health grant AR 33667.

1 Packer S, Rotman M, Salanitro P. Iodine-125 irradiation of choroidal melanoma, clinical experience. Ophthalmology 1984; 91: 1700-8.

2 Lommatzsch P. b Irradiation of choroidal melanoma with ${ }^{106} \mathrm{Ru} /{ }^{106} \mathrm{Rh}$ applicators. Arch Ophthalmol 1983; 101: 713-7.

3 Augsberger JJ, Gamel JW, Sandi VF. Enucleation vs. cobal plaque radiotherapy for malignant melanomas of the choroid and ciliary body. Arch Ophthalmol 1986; 104: 655-9.

4 Last RJ. Wolff's anatomy of the eye and orbit. Philadelphia: Saunders, 1970: 326.

5 Kline RW, Yeakel PD. Ocular melanoma, I-125 plaques. Med Phys 1987; 14: 475 .

6 Hendrix LE, Massaro BM, Daniels DL, Smith DF, Haughton VM. Surface coil MR evaluation of a lacrimal gland VM. Surface coil MR evaluation of a lacrimal
carcinoma. F Comput Assist Tomogr 1988; 12: 866-8.

7 Earle J, Kline RW, Robertson DM. Selection of iodine-125 for the Collaborative Ocular Melanoma Study. Arch Ophthalmo 1987; 105: 763-4. 
8 Packer S, Rotman M. Radiotherapy of choroidal melanoma with iodine-125. Ophthalmology 1980; 87: 582-90.

with iodine-125. Ophthalmology 1980; 87: 582-90. Chan B. Irradiation of choroidal melanoma with iodine-125 0 Fried M, Foerster MH, Wessing A, Meyer-Schwickerath Echographische Grossenbestimmung von Tumor und
Lageuberprufung des Applikators in der RutheniumLageuberprufung des Applikators in der Ruthenium-
therapie beim Aderhautmelanom. Fortschr Ophthalmol

11 Williams DF, Mieler WF, Lewandowski M, Greenberg M Echographic verification of radioactive plaque position in the treatment of melanomas. Arch Ophthalmol 1988; 106 $1623-4$ 